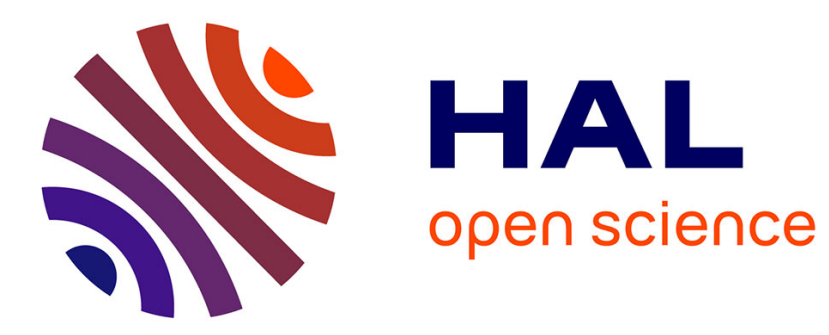

\title{
Conceptual (and hence mathematical) explanation, conceptual grounding and proof
}

\author{
Francesca Poggiolesi, Francesco A Genco
}

\section{To cite this version:}

Francesca Poggiolesi, Francesco A Genco. Conceptual (and hence mathematical) explanation, conceptual grounding and proof. Erkenntnis, In press. hal-03176283

\section{HAL Id: hal-03176283 \\ https://hal.science/hal-03176283}

Submitted on 22 Mar 2021

HAL is a multi-disciplinary open access archive for the deposit and dissemination of scientific research documents, whether they are published or not. The documents may come from teaching and research institutions in France or abroad, or from public or private research centers.
L'archive ouverte pluridisciplinaire HAL, est destinée au dépôt et à la diffusion de documents scientifiques de niveau recherche, publiés ou non, émanant des établissements d'enseignement et de recherche français ou étrangers, des laboratoires publics ou privés. 


\title{
Francesca Poggiolesi, Francesco Genco \\ Conceptual (and hence mathematical) ex- planation, conceptual grounding and proof
}

\begin{abstract}
This paper studies the notions of conceptual grounding and conceptual explanation (which includes the notion of mathematical explanation), with an aim of clarifying the links between them. On the one hand, it analyses complex examples of these two notions that bring to the fore features that are easily overlooked otherwise. On the other hand, it provides a formal framework for modeling both conceptual grounding and conceptual explanation, based on the concept of proof. Inspiration and analogies are drawn with the recent research in metaphysics on the pair metaphysical grounding-metaphysical explanation, and especially with the literature in philosophy of science on the pair causality-causal explanation.
\end{abstract}

\section{Introduction}

Metaphysics and philosophy of science are traditionally two separate branches of philosophy which develop and express themselves in ways that are often quite different. However, one interesting parallel between them involves pairs of kindred notions that cover a central role in each: the pair metaphysical grounding-metaphysical explanation, which has recently gained attention and became the focus of metaphysical studies, ${ }^{1}$ and the dichotomy causality-causal explanation in philosophy of science. ${ }^{2}$

The relationship involved in each - i.e. that between metaphysical grounding and metaphysical explanation on the one hand, and that between causality and causal explanation on the other - is or has been dealt with in the respective disciplines. For each discipline, however, the focus is different. In metaphysics, a fairly recent and active debate ${ }^{3}$ concerns the identity, or otherwise, of the notions of metaphysical grounding and metaphysical explanation. According to the unionist point of view, metaphysical explanation and metaphysical grounding coincide, whilst according to the separatist point of view, metaphysical grounding and metaphysical explanation come apart, the former notion backing the latter. ${ }^{4}$ By contrast, in philosophy of science, there has been much emphasis on the structures of causality and causal explanation. ${ }^{5}$ Given the relevance of more complex examples of causal explanations coming from sciences, there is a

\footnotetext{
${ }^{1}$ E.g. see Correia and Schnieder (2012).

${ }^{2}$ E.g. see Menzies and Beebee (2019); Woodward (2019).

${ }^{3}$ E.g. see Glaizer (2020); Maurin (2019).

${ }^{4}$ See Raven (2012).

${ }^{5}$ See Hempel (1942); Woodward (2003).
} 
natural need to understand the structure of these examples and such structures then illuminate the link with the notion of causality. Hence, the relationship causality-causal explanation is studied from a structuralist angle.

More recent research ${ }^{6}$ has seen the rise of the idea that grounding and explanation might not only be significant notions from a metaphysical perspective, but they can also reveal particular connections between the sentences of our language via the meaning that these sentences express, or via the concepts occurring in them. These are cases of so-called conceptual grounding and conceptual explanation. According to Smithson (2020, p. 4), typical examples of conceptual grounding are:

(a) The wall is red because it is scarlet,

(b) John is a bachelor because he is a man and he is unmarried,

(c) that animal is a vixen because that animal is a fox and it is a female.

According to Schnieder (2006, p.403), typical examples of conceptual explanations are:

(d) Federica is my sister-in-law because she is married to my brother,

(e) Xanthippe is a widow because Socrates died,

(f) this vase is colored because it is red.

It seems natural to extend the parallel between the causality-causal explanation and metaphysical grounding-metaphysical explanation pairs to this new pair consisting of conceptual grounding and conceptual explanation. However, as we have seen above, this issue can be approached from two interconnected yet different perspectives, that adopted by metaphysics or that prevalent in philosophy of science. Since, as we will show later, the pair conceptual groundingconceptual explanations involves cases coming from mathematics which display a complexity analogous to examples considered in philosophy of science, the aim of this paper is to take some first steps towards clarifying the relation between conceptual grounding and conceptual explanation by adopting a structuralist point of view: the link between these two notions will be studied by focussing on the structures the underlie them. To do this, we will use and rely on the separate and historically well-established literatures on conceptual groundingconceptual explanation on the one hand, and philosophy of science on the other.

The paper is organized in the following way. In Section 2, we will introduce the notions of conceptual grounding and conceptual explanation and we will argue that, although perhaps slightly overlooked in the literature to date, the notions of conceptual grounding and conceptual explanation apply not only to simple toy examples, such as those above, but also to some more complex examples coming from mathematics. In Section 3 we shall study the relationship between conceptual grounding and conceptual explanation, paying particular

${ }^{6}$ E.g. see Betti (2010); Chalmers (2012); Schnieder (2006); Smithson (2020). 
attention to more complex examples, which, as in the causal case, highlight characteristics of the relationship that are out of focus on simpler examples. In Section 4, we will elaborate a solid formal framework for modeling the different structures behind the notion of conceptual explanation and conceptual grounding, which have emerged in the previous section. For this goal will use the notion of proof, that independent studies have linked both to the notion of conceptual grounding as well as to the notion of conceptual explanation. ${ }^{7}$. Finally, in Section 5 we will draw some conclusions.

\section{Conceptual grounding and conceptual expla- nation}

We begin by introducing the notions of conceptual grounding and conceptual explanation, as they are usually treated in the contemporary literature. This introduction will not only serve to better understand these notions, but also to provide examples which will be useful later in the paper.

\subsection{Conceptual grounding}

In the last two decades, philosophers have focussed on the notion of metaphysical grounding, which is often described as a relation amongst facts (or truths) that is objective, non-causal and explanatory in nature. Metaphysical grounding relates items on different ontological levels and it concerns the structure of the world. Beside the notion of metaphysical grounding, the notion of conceptual grounding has recently gained some attention; conceptual grounding is a relation that creates connections between different semantic levels, as (Smithson, 2020, p. 3) puts it, or between different conceptual levels, as (Betti, 2010, p. 284) understands it. ${ }^{8}$ These connections could be of several kinds ${ }^{9}$ and they support the relation between ground(s) and consequence.

There are two opposite ways to look at the links between metaphysical and conceptual grounding. On the one hand, Correia (2014) explicitly formulates a view, which seems to be shared by other scholars, ${ }^{10}$ that consists in taking conceptual grounding as part of metaphysical grounding: any case of conceptual grounding is also a case of metaphysical grounding, while the opposite does not hold. On the other hand, a rising number of philosophers ${ }^{11}$ draw a sharp

\footnotetext{
${ }^{7}$ On the links between conceptual grounding and proof-theory see Poggiolesi (2018); Prawitz (2019); Rumberg (2013). On the links between mathematical explanations and the notion of proof see Mancosu (1999, 2001).

${ }^{8}$ Note that the difference between Smithson's conception and Betti's conception is marginal for the discussion here, and therefore through the paper we will equivalently either refer to one or the other. As (Smithson, 2020, p. 2) says: "It would be interesting to consider which type of conceptual grounding is comparatively fundamental. This question may be related to the dispute over the relative primacy of mental representation vs. public language meaning."

${ }^{9}$ See (Smithson, 2020, p. 4).

${ }^{10}$ E.g. Fine (2012); Raven (2015).

${ }^{11}$ E.g. see Audi (2012); Betti (2010); Hofweber (2009); Merlo (2020); Smithson (2020).
} 
division between metaphysical and conceptual grounding: the two notions come apart and a genuinely explanatory because-claim expresses either metaphysical grounding or conceptual grounding.

In this paper we avoid taking sides in this debate and elaborate an approach to conceptual grounding compatible with both positions. In practice, this means treating conceptual grounding as a sui generis notion. There are independent reasons for doing so, most notable of which is the oft-neglected feature that conceptual grounding not only encompasses logical grounding, ${ }^{12}$ but also what we might call mathematical grounding, ${ }^{13}$ namely grounding that connects sentences in virtue of mathematical properties. Consider, for example, the following two sentences $p$ and $q$ :

$p$ : given two points $a$ and $b$, there is a third point $c$ such that $c a=c b=a b$,

$q$ : given two circles, one described around the center $a$, the other around $b$, both with a radius $a b$ and lying in one and the same plain, these circles always intersects in a point $c$ such that $c a=c b=a b$.

Let us accept that the claim ' $q$ because $p$ ' is a grounding claim; ${ }^{14}$ then one must also accept that ' $q$ because $p$ ' is a conceptual grounding claim since it connects sentences in virtue of the (mathematical) concepts (or meaning of terms) involved in them. This position is shared and put forward by a milestone in the study of grounding, namely Bernard Bolzano. Bolzano focussed on the notion of conceptual grounding, as a relation holding amongst conceptual truths ${ }^{15}$ that is irreflexive, asymmetric and antitransitive. ${ }^{16}$ Since mathematical truths belong to the realm of conceptual truths, in Bolzano's Theory of Science many examples of conceptual grounding are actually examples of mathematical grounding, like the one above. Indeed, the inclusion of such cases in the domain of conceptual grounding indicates the breadth of its scope, from the simple examples of the Introduction, reminiscent of those discussed in the metaphysical literature, to more refined ones with strong analogies to those debated in the literature on causality. As we will try to show, these mathematical examples constitute a paradigmatic and instructive case study, shedding light on the distinctive characteristics of conceptual grounding.

\subsection{Conceptual explanation}

The notion of explanation is one of the most important in philosophy; in the philosophy of science it has given rise to a wide literature, which, due to the emphasis on natural sciences tends to centre on causal explanation. In present-day analytic metaphysics it has also received renewed attention; the debate makes

\footnotetext{
${ }^{12}$ This has already been observed by Correia (2014).

${ }^{13}$ Mathematical grounding as a subtype of conceptual grounding is also considered in Carrara and De Florio (2020).

${ }^{14}$ See for example (Bolzano, 2004, §13).

${ }^{15}$ The notion of conceptual grounding in Bolzano's philosophy involves differences with the modern conception, e.g. see Roski and Schnieder (2019); Rumberg (2013).

${ }^{16}$ E.g. see Tatzel (2002).
} 
appeal to the notion of metaphysical explanation that philosophy of science, by contrast, slightly marginalizes. This paper is concerned with yet another type of explanation, namely conceptual explanation.

To the best of our knowledge, conceptual explanations have so far only been treated by Betti (2010); Schnieder (2006). As (Betti, 2010, p. 283) says, it is not easy to rigorously characterize them: they can be seen as explanations connecting sentences on the basis of the links, which might be of several kinds, ${ }^{17}$ amongst concepts occurring in them, or of the meaning of expressions involved in the sentences themselves. In particular, following (Schnieder, 2006, p. 404), we can distinguish between a subjective and an objective notion of explanation. An explanation in the first sense is just a piece of information enlarging our understanding of a given matter; for example, if one explains how addition works to a child by providing several examples of addition, then one is giving an example of subjective explanation. Explanations in the subjective sense represent a 'loose' way of talking about explanations. ${ }^{18}$

On the other hand, an explanation is objective when it involves (or it is) an objective relation between explanans and explanandum. Consider for example the relation between the cigarette lit in the forest and the fire in the forest: this relation is causal in nature and holds independently of what a particular subject might think of it. An explanation that takes into account (or amounts to) such a relation, like the following one:

- there is a fire in the forest because a cigarette was lit in the forest,

is an objective causal explanation since it is (based on) an objective relation that is causal in nature. Relations with these characteristics can occur not just in virtue of a causal link between facts (or events), but also in virtue of a conceptual link, carried by the concepts or due to the meanings of the expressions involved. Consider the concept (or linguistic expression) (i) "sister-in law of $x$," as well as the concepts (or linguistic expressions) (ii) "wife of the brother of $x$." There is evidently a link between (i) and (ii): it is a link of conceptual or semantic analysis $^{19}$ (e.g. when we analyze the concept or expression 'sister-in law of $x$ ' we end up with "wife of the brother of $x$ ") that is non-causal and objective in nature. Hence, an explanation that takes into account (or amounts to) this link, such as the conceptual explanation (d):

- Federica is my sister-in-law because she is the wife of my brother

is a conceptual explanation since it is (based on) an objective relation which is conceptual or semantic in nature. As (Schnieder, 2006, p. 404) clearly sums up while discussing explanations, conceptual explanations hold in virtue of objective relations amongst concepts and independently of the informational background of a person confronted with them. However, these explanations retain

\footnotetext{
${ }^{17}$ See (Schnieder, 2006, p. 405).

${ }^{18}$ See (Schnieder, 2006, p. 404).

${ }^{19}$ Conceptual explanations presuppose these conceptual-semantic-links. Hence, those philosophers, as Williamson (2007), who are altogether skeptical of such conceptual-semantic links, will be arguably skeptical of conceptual explanations.
} 
their epistemological force and status, which is that of convincing of the truth of the explanandum. There are even illustrious philosophers, as (Aristotle, 1984, 115, Post. An., 71b 20-25) or (Bolzano, 1996, p.178), for whom the kind of explanations revealing the objective grounds of the explananda are those explanations that in the easiest and most constructive way secure the conviction of the truth of their explananda. Hence in these explanations the ontological and the epistemological levels seem to go hand in hand.

A closely related family of explanations are mathematical explanations, which in the contemporary literature were firstly studied by Steiner (1978) and then analyzed by Kitcher (1981) and are today a wide and important field of research (e.g. see Mancosu $(2001,2008)$ ). Standardly, they are divided into internal and external mathematical explanations. ${ }^{20}$ For reasons that will become evident shortly, we focus on the former. Internal mathematical explanations are explanations within pure mathematics, namely proofs of mathematical results that involve only other mathematical results. For example, there are many different proofs of Pythagoras's theorem, but one of them is standardly considered as an explanation of Pythagoras's theorem itself: this proof provides the reasons why the theorem is true. These reasons are objective - they do not depend on what someone might think of them - and non-causal in nature - since casual relations hold amongst empirical facts but not amongst mathematical ones. (Schnieder, 2006, footnote 20) and Betti (2010) agree in considering this explanation of Pythagoras's theorem, as all internal mathematical explanations that share the same characteristics, as conceptual explanations; as Betti says: ${ }^{21}$

Explanation in mathematics and conceptual explanation in presentday metaphysics do not just cross paths: they are one of a kind.

(Betti, 2010, p. 252)

Precisely because conceptual explanations include mathematical explanations which may display a high level of complexity, the question of the structure underlying and thus clarifying these explanations is of particular importance. Certainly, Bernard Bolzano felt this importance, since, as Mancosu (2008) stresses, he could be seen as providing the first full systematization of conceptual, and hence also mathematical, explanations. Following his lesson, we will try to provide a novel structural analysis of conceptual explanations; this analysis will also clarify the relation with the notion of conceptual grounding.

\footnotetext{
${ }^{20}$ External mathematical explanations are explanations in which non-mathematical phenomena are partially explained by mathematical findings e.g. the explanation of why hive-bee honeycombs have a hexagonal structure in virtue of the fact that any partition of the plane into regions of equal area has as perimeter at least that of the regular hexagonal honeycomb tiling.

${ }^{21}$ According to Betti, even external mathematical explanations should count as conceptual explanations. However since in this case the link might be more controversial and Schnieder does not mention it, we leave this type of explanations aside for future research.
} 


\section{On the relationship between conceptual ground- ing and conceptual explanation}

We have introduced two notions, conceptual grounding and conceptual explanation, that even at the first glance seem to have a tight connection. We use this section to precisely spell out their relationship.

\subsection{Common points between conceptual grounding and conceptual explanations}

The existing literature on conceptual grounding and conceptual explanation, which we reviewed in the previous section, brings up the following common points. First of all, both notions connect sentences; secondly, both notions connect sentences in virtue of the concepts or meaning of expressions contained in the sentences themselves; thirdly, both notions include a mathematical sphere which was noticed by Bolzano but is often neglected in some branches of the contemporary literature.

A fourth common point concerns a notion that, though not mentioned yet, is frequently retained in the existing literature as central to both conceptual grounding and conceptual explanation: complexity. Let us consider grounding and explanation in turn.

In the literature on conceptual grounding, complexity is generally taken to play a role analogous to that played by fundamentality in the literature on metaphysical grounding. Scholars working on metaphysical grounding standardly assume $^{22}$ it to be a relation between more fundamental and less fundamental truths or facts. Grounding is the mortar relating different levels in a hierarchical edifice in which the less fundamental rest on the more fundamental. In the literature on conceptual grounding, the picture is that of an analogous hierarchy, but where levels are differentiated by their conceptual complexity. Conceptual grounding is the mortar connecting conceptually more complex consequences with their less complex grounds.

The classic statement of this vision, which has informed work on conceptual grounding since, is due to Bolzano. A cornerstone of his theory of Abfolge is the role of complexity as one of the main features of the notion of conceptual grounding. According to him, it is possible to isolate a certain domain, that of conceptual truths, in which grounding induces a reduction of a particular sort of complexity, namely, conceptual complexity: (conceptual) grounds are conceptually less complex than their consequences. In particular, when descending from a conceptual truth to its grounds, a level where no further reduction is possible will be eventually reached. This is the base level in which all conceptual truths are grounded, and its characterizing feature is simplicity. ${ }^{23}$

\footnotetext{
${ }^{22}$ E.g. see Audi (2012); Raven (2015).

${ }^{23} \mathrm{~A}$ detailed reconstruction of Bolzano's notion of conceptual complexity can be found in Roski and Rumberg (2016); Roski and Schnieder (2019).
} 
Like the notion of fundamentality in the literature on metaphysical grounding, ${ }^{24}$ conceptual complexity is often taken to be unanalyzed and intuitive; accordingly, detailed study of the notion in its full generality is scant. That said, some elements of analytical rigor have been proposed, at least for certain cases. For instance, for logical grounding, which is a case of conceptual grounding, a clear and fine-grained notion of logical complexity has been defined; ${ }^{25}$ this notion adequately tracks complexity increases from logical grounds to their conclusion. Or, to take another example, there are concepts that can be easily defined in terms of other concepts: to the extent that the former can be seen as composed from the latter, they count as more complex. ${ }^{26}$ The concept of bachelor, say, can be defined in terms of the concept of man and the concept of unmarried, and hence can be seen as composed by the concept of man and that of unmarried and therefore it should count as more complex than the concepts of man and unmarried. Or, to take another example, the color red can be defined as the set of all types of red - crimson, scarlet, .. - and hence can be seen as composed of them. In this case, the color red will count as more complex than the color crimson. ${ }^{27}$ Although such examples most likely do not cover all cases of conceptual grounding, ${ }^{28}$ they provide some further clarification into the notion of conceptual complexity. For the purposes of this paper, we can follow the bulk of this literature in taking conceptual complexity as an unanalyzed notion, recognizing that there may be cases where there is more piercing understanding to be had.

In the literature on conceptual explanation, conceptual complexity is equally central. A point of agreement between Betti (2010) and Schnieder (2011) is that relations of conceptual complexity, which are independent of any epistemic subject, are a constitutive part of conceptual explanations: these explanations can indeed be seen as chains of sentences where complexity increases from the explanans to the explanandum. Conceptual explanations are, like any other type of explanation, asymmetric, but in the case of conceptual explanations this asymmetry is due to factors of conceptual complexity and primitiveness: sentences containing more complex concepts are explained by sentences containing simpler ones. Moreover, in mathematical explanations, which are a particular type of conceptual explanation, the issue of complexity increase from explanans to explicandum has often been evoked. According to Detlefsen (1988) and Paoli (1991), there is a long list of thinkers in the philosophy of mathematics, including Arnauld and Nicole (1970); Bolzano (2015); Frege (2016); Leibniz (1996); Pascal (1993), who conceive the truths of mathematics as divided in an objective hierarchy of simpler and more complex truths. Explaining a mathematical truth amounts to revealing its place in such a hierarchy and thus connecting it

\footnotetext{
${ }^{24}$ What we call here fundamentality corresponds to what Tahko (2018) calls relative fundamentality. As he says "Given its importance, it is perhaps surprising that there are relatively few explicit accounts of relative fundamentality in the literature so far."

${ }^{25}$ See Poggiolesi (2016).

${ }^{26}$ See Ginammi et al. (2020).

${ }^{27}$ In Section 4, we will put forward an alternative conceptual complexity scale for the colors red and crimson.

${ }^{28}$ See Ginammi et al. (2020).
} 
with simpler truths that are the reasons behind it.

In summary, conceptual complexity is widely considered to be a main feature of conceptual grounding and conceptual explanation in the literatures on these topics, much as fundamentality is typically thought of as a main feature of metaphysical grounding and metaphysical explanation in the literature on them. Moreover, conceptual complexity has illustrious roots and is linked to the notion of logical complexity as well as to a hierarchical conception of mathematics. The directionality of conceptual grounding and conceptual explanation can be seen as the result of the relation of conceptual complexity that characterizes these notions.

Given all these similarities between conceptual grounding and conceptual explanation, one might reasonably wonder whether these two notions are the same. Indeed, if we look at the typical examples of conceptual grounding and explanation in the literature (see for instance those in the Introduction), they are so similar that it is hard to specify why any example represents one notion and not the other. Consider the following paradigmatic example of conceptual grounding: "John is a bachelor because John is a man and John is unmarried." According to what has been said so far, is there any reason for it not to also count as an example of conceptual explanation? Consider, on the other hand, the following typical example of conceptual explanation: "Xanthippe is a widow because Socrates died." Is there any reason for it not to also count as an example of conceptual grounding? The temptation to conclude that conceptual grounding and conceptual explanation are indeed one and the same notion is strong. However, as we will now argue, a deeper study reveals that this temptation should be resisted: they are different in subtle, yet important ways.

\subsection{More complex examples: the causality-causal expla- nation case}

So far we have studied the link between conceptual grounding-conceptual explanations only under the light of mere platitudes, like those concerning the concepts of bachelor and widow. However both notions subsume a mathematical sphere rich with more intricate examples. As philosophy of science teaches us, these examples should be also taken into account, probably being the most enlightening. This will indeed be our strategy in what follows. However, in order to clarify our argument, we will first introduce a paradigmatic complex example of causality and causal explanation and briefly summarize the literature concerning it. In the next section we will move to the conceptual level and draw on the observations made with respect to causality and causal explanations.

Scientists by now agree that there is a causal relation between fridges, air conditioners and climate change. So scientists will be willing to accept the following sentence

(1) "there are climate damages (partly) because we use fridges and air conditioners" 
as a true sentence that conveys a relation, that is causal in nature, between the use of some objects and world-wide well-known climate situation. We, nonscientists, might also be willing to believe such sentence to be true, trusting scientists, and hence to accept that there is a causal relation between fridges, air conditioners and climate change. The question now arises: would sentence (1) count as a genuine, albeit partial, causal explanation of climate change? The answer seems to depend on the what one takes to be the aim or the point of providing an explanation. If we take explanations to show why a phenomena occurs $^{29}$, i.e. if we take explanations to show that a certain phenomenon is to be expected, then (1) cannot count as an explanation since nobody would expect climate change by simply evoking fridges and air-conditioners (this has actually been the case for decades). Sentence (1) does not make anybody understand how fridges and air conditioners cause climate change: it conveys a relation between two state of affairs but in no way clarifies how and why this relation holds. In other words, sentence (1) states a link between fridges, air conditioners and climate damages, but it doesn't show how the explanandum-phenomenon is to be expected and thus it does not satisfy the necessary condition of providing understanding. So sentence (1) fails to count as an explanation.

In the scientific explanation literature there seems to be a wide consensus ${ }^{30}$ about what needs to be added to (1) in order for it to become an explanation. Laws or, more generally, generalizations are the missing ingredients; in order for (1) to be an explanation, we need to specify which generalizations connect fridges and air conditioning to climate change. This view is shared by many different approaches: by the Deductive-Nomological model put forward by Hempel (1942), by the Unionist account proposed by Kitcher (1981), but also by the Counterfactual account defended by Woodward (2003). The main difference between these approaches does not concern the importance of laws or generalizations in explanations but their role in the structure of explanations (the role will be important in this paper as well, as discussed in the next Section). For example, according to the DN-model (1) would become an explanation if it was rephrased according to the following structure:

$$
\begin{aligned}
& \mathrm{S} \text { and } \mathrm{K}_{1}, \ldots \mathrm{K}_{n} \text {, then } T \\
& \mathrm{~S} \text { and } \mathrm{K}_{1}, \ldots \mathrm{K}_{n}
\end{aligned}
$$

$\mathrm{T}$

where $\mathrm{S}=$ the fact that we use our fridges and air conditioners, $\mathrm{T}=$ climate damages, $\mathrm{K}_{1}$ = fridges and air conditioners work if, and only if, electricity (as fossil fuel) is produced; $\mathrm{K}_{2}=$ the law of combustion (which says that electricity - produced as fossil fuel - causes the emissions of $\left.\mathrm{CO}_{2}\right) ; \mathrm{K}_{3}, \ldots \mathrm{K}_{n}$ = physical and chemical laws linking emission of $\mathrm{CO}_{2}$ to climate damages.

\footnotetext{
${ }^{29}$ This seems a widespread conception behind explanations, see (Woodward, 2003, p. 153).

${ }^{30}$ E.g. see Hempel (1942); Jansson (2017); Salmon (1984); Strevens (2008); Woodward (2003).
} 
Why is (1a) to be considered as an explanation? More generally, why do so many philosophers of science require the presence of laws or generalizations to talk about explanations? The literature on this issue is immense and we could not treat it in any detail here. However, three main lessons seem to be recurrent.

First of all, as mentioned above, laws or generalizations are necessary in explanations since they allow the occurrence of the phenomenon explained to be expected and in this sense they contribute to making us understand why the phenomenon occurred. This is one of Hempel's main ideas and what Salmon (1984) calls the nomic expectability, namely expectability on the basis of laws or generalizations. Secondly, the requirement of laws or generalizations for explanations is supported and justified by a Humean conception of the nature of causality. According to this conception, causality cannot be taken as primitive

and the holding of regularities that support causal claims is a non-circular way of preventing such causal claims to remain without analysis and elucidation. But precisely because generalizations or laws provide an elucidation for causal claims, then it is reasonable to argue that they contribute to explanations in which causal claims occur. Thirdly and finally, the presence of laws or generalizations in scientific explanations fits very well with the scientific practice: in many areas of science, e.g. physics, chemistry, but also economics and evolutionary biology, scientists use laws or generalizations to explain phenomena. "Explaining various macroscopic electromagnetic phenomena typically involves writing down and solving one or more of Maxwell's equations" (Woodward, 2003, p. 185), in the same way "explaining some elementary quantum-mechanical phenomenon will involve modeling some physical systems in such a way that we can apply the Schrödinger equation to it," (Woodward, 2003, p. 185). Since the use of laws or generalizations is a pervasive characteristic of explanatory practice, a theory of explanation must acknowledge it.

In virtue of what has been said up to now, we can think of the difference between causality and causal explanation as boiling down to this: whilst causality is merely a (binary) relation between facts or events (the cause(s) and the effect), a causal explanation is a structure - which varies from approach to approach - including an explanans, an explanandum and the generalization(s) or law(s) that link the two. In other words, the upshot of this literature is that causal claims miss something that prevent them from being bona fide explanations, and that thing is generalizations. However, causal claims do posess an explanatory force which is provided by their proximity to causal explanations.

\subsection{More complex examples: the conceptual grounding- conceptual explanation case}

Turning now to conceptual grounding and conceptual explanation, let us begin with a complex example that concerns both. The example comes from mathematics: as we have said, the mathematical sphere concerns both concep- 
tual grounding and explanation. According to a (largely) Bolzanian analysis, ${ }^{31}$ there exists a non-causal explanatory relation connecting the equality between the sum of three angles of any triangle and two right angles on the one hand, and the equality between the sum of the four angles of any quadrangle and a full circle on the other. So Bolzano would be willing to accept the following sentence:

(2) "the sum of the four angles of every quadrangle is equal to a full circle because the sum of the three angles of a triangle is equal to two right angles"

as a true sentence conveying a relation, that is non-causal and explanatory in nature, between the angles of any triangle and the angles of any quadrangle. For the purposes of this example, let us assume, trusting Bolzano, that there is indeed a mathematical, and hence conceptual, grounding relation between the angles of triangles and the angles of quadrangles. The question now arises: would sentence (2) count as a genuine conceptual explanation of why the sum of the four angles of every quadrangle is equal to a full circle? If we adapt the nomic-expectability perspective discussed above to the conceptual case - namely if we think that the point of providing a conceptual explanation is to show that a certain item is to be expected to be true, then (2) cannot be an explanation since by simply evoking the sum of the three angles of a triangle, one would not expect that the fact that the sum of the four angles of every quadrangle is equal to a full circle is true. In other words, sentence (2) does not make anybody understand how the sum of the angles of a triangle can determine a feature of the sum of the angles of a quadrangle: it conveys a relation between triangles and quadrangles but in no way clarifies how or why this relation holds. Sentence (2) states a link between triangles and quadrangles, but it doesn't show how the explanandum is to be expected to be true and thus it does not satisfy the necessary condition of providing understanding. So sentence (2) fails to count as an explanation.

Our idea is that, in order for sentence (2) to become an explanation, the non-causal relation between triangles and quadrangles needs to be accompanied by the generalization(s) underlying this relation. More generally, we want to put forward an approach based on the claim that just like causal explanations, conceptual explanations, in order to be explanations, need an explicit reference to or use of generalizations. Let us first defend this claim; then we will show what the structure of conceptual explanations is, according to our approach. In order to defend the necessity of generalizations in conceptual explanations, we will use and adapt to the conceptual context the three main arguments previously cited in the causal context.

First of all, as mentioned above, generalizations are necessary in explanations since they allow the explanandum to be expected to be true and in this sense they contribute in making us understand why the explanandum is true.

\footnotetext{
${ }^{31}$ We have slightly changed Bolzano (2004)'s example to make it more adequate for our study.
} 
Hempel's nomic expectability seems easily adaptable to the conceptual context: expecting a sentence to be true on the basis of generalizations contribute the one's understanding of why the sentence is true. Therefore, generalizations are necessary for explanations in order for them to satisfy their epistemological aim.

The second argument used in the causal context appealed to a Humean conception of causality. Recall that under this conception, causality is not primitive, and the central role of laws in supporting causal claims underpins the importance of laws for causal explanation. Clearly, there is no strict analogue to the Humean conception of causality for grounding; however, there do exist accounts of conceptual grounding that do not treat it as unanalyzable, the most notable of which was proposed by Bolzano. Indeed, one trend of the current literature on grounding ${ }^{32}$ precisely takes up this Bolzanian perspective and attempts to provide a definition of grounding in terms of derivability and complexity. ${ }^{33}$ The following picture emerges from this strand of research. For conceptual grounding, the starting point are generalizations, notably in the form of definitions, logical rules, mathematical theorems, conventions and so on (we will return to this point later). These generalizations fix the relations among concepts and hence serve to build the hierarchy of conceptual complexity mentioned above, in which concepts and conceptual truths are classified. As concerns grounding, it serves to reveal connections amongst conceptual truths of different levels of the hierarchy, in that (sentences conveying) less complex conceptual truths ground (sentences conveying) more complex ones. In this picture, it is the generalizations that support the hierarchy behind grounding claims, much as laws support causal claims under the Humean approach to causality. Hence generalizations represent a non-circular way for providing an analysis of grounding claims. But precisely because generalizations give an elucidation for grounding claims, then, just as for the case of causal explanations, it is reasonable to argue that generalizations contribute to explanations in which grounding claims occur. Hence generalizations are a necessary element of conceptual explanations.

Let us now move to the third and final argument in defense of the presence of generalizations in conceptual explanations; such presence fits very well with the scientific practice. As already said, conceptual explanations include mathematical explanations and a mathematical explanation is typically a proof that show why the theorem is true by evoking a general mathematical property or a mathematical definition. ${ }^{34}$ For example the proof that explains why Pythagora's theorem is true uses a general mathematical property which says that the areas of similar plane figures are to each other as the squares of the corresponding sides. Hence, since the use of generalizations is a characteristic of the mathematical explanatory practice, a theory of explanation that covers inter alia mathematical explanations must acknowledge it.

Having defended the use of generalizations in conceptual explanations, let us turn to the role that generalizations need to have in explanations, according to our approach. We want to put forward the idea that generalizations are not,

\footnotetext{
${ }^{32}$ E.g. see Poggiolesi (2016).

${ }^{33}$ E.g. see also Ginammi et al. (2020).

${ }^{34}$ E.g. see Mancosu (1999); Steiner (1978).
} 
as in the DN-model, part of the explanans, but they represent what connects and supports the link between explanans and explanandum. To precisely fit this role, if the relation between explanans and explanandum is mediate, it needs to be decomposed in steps in such a way that each step is supported by the relevant generalization. Let us illustrate this point, which will also be developed in the next section, by showing how, according to our view, sentence (2) needs to be changed in order to become a bone fide explanation. This is our proposal:

(2a) The reason why the sum of the four angles of any quadrangle is equal to a full circle is that the sum of the four angles of any quadrangle is equal to four right angles, via definition (i) that says that any full circle corresponds to four right angles. The reason why the sum of four angles of any quadrangle is equal to four right angles is that the sum of the three angles of a triangle is equal to two right angles, via theorem (ii) that says that every quadrangle can be divided into two triangles whose angles taken together are equal to the angles of the quadrangle.

Let us dwell on paragraph (2a). It is easy to note that (2a) still connects triangles and quadrangles but, in addition, (I) it specifies which steps are needed to go from one to the other, (II) it justifies each step with a generalization, which, in this example, takes the form of a theorem (see (ii)), or a definition (see (i)). So in (2a) we still have an explanans and explanandum as in (2) (although in (2) they are standardly called ground and consequence, respectively), but we also have the structure that links the two, made of steps together with the generalizations supporting each step. In (2a) the presence of generalizations that are needed in explanations is ensured as in the causal context. However, in $(2 \mathrm{a})$ the role of generalizations in explanations is novel: generalizations are the connections relating the explanans to the explanandum and this is why we need to divide the conceptual explanation in steps since we need to say exactly which explanandum and explanans each generalization links. This way of treating generalizations not only has several technical advantages that will be illustrated in the next section, but is also novel and original. Moreover, it respects and reflects the general picture which arises from the previous discussion. As we have said, the holding of generalizations is what supports grounding claims; this is also true in the causal context where laws support causal claims. However, whilst the DN-model, say, betrays this role of generalizations since in the DN-model generalizations are part of the explanans, our approach faithfully systemizes this idea: according to our approach, the structure of conceptual explanations is such that generalizations have a different status from the explanans (i.e. ground) and the explanandum (i.e. consequence), they are neither part of one nor of the other, they are what links the two. In the next section, we will provide this idea with a proper formalization that will help clarifying it and add further arguments for it.

We have so far focussed on conceptual explanations, defending the need of generalizations in conceptual explanations as well as a particular use of such 
generalizations. We have not said much about conceptual grounding or its structure. However, as far as we can see, at this point, there are two possibilities: either we take conceptual grounding to have the same structure as conceptual explanations, or we do not. We see two important arguments for the second option. First of all, because it is a widespread claim of the current literature that grounding, be it metaphysical, conceptual or logical, is a relation, not a structure, amongst items of different sorts. Secondly, because grounding runs on a strict parallel with causality ${ }^{35}$ and causality is taken to be a relation between cause and effect. Hence it seems fair to conclude that while a conceptual explanation is a structure that starts with (an) explanans - the ground(s) - ends with an explanandum - the consequence - and specifies the steps that link the two by displaying the relevant generalization(s), which is a definition, or a logical rule, or a convention, or a mathematical theorem and so on, conceptual grounding is a (binary) relation between sentences (ground(s) and consequence), holding in virtue of the concepts or the meaning of expressions contained in them. Note that according to this picture conceptual grounding comes close to conceptual explanation: it has all the elements to count as one; simply, it does not require the use of generalizations. Hence, according to this perspective, it sounds very natural to look at conceptual grounding as a relation having an explanatory force although falling short of a bona fide explanation. The formalism that will be introduced in the next section will serve to precisely spell out the relationships between conceptual grounding as a relation and conceptual explanation as a structure of a particular type.

\subsection{General remarks}

Let us conclude this section by offering some general remarks about the conclusions that have just been drawn. The first concerns the use of generalizations in conceptual explanations. As (Jansson, 2017, p. 10) notes, in the case of causal explanations the difference between laws or generalizations and accidents is often treated as a given in the analysis: it is possible to gain insights in the phenomenon of causal explanations without necessarily studying these related notions. In particular, we can take the fact that some generalizations have a special role in causal explanations as a given, as long as we think of these explanations as relative to some theory $T$; under this perspective, it is the theory $T$ that determines what should be considered as a generalization and thus treated as pertaining to a different level.

In the present paper, we adopt this methodological strategy for conceptual explanations. Therefore we will treat the difference between generalizations in the sense of definitions, theorems, conventions, and so on - and accidents as a given in the analysis. In other words, we consider a notion of conceptual explanation, and a related notion of conceptual grounding, as relative to a theory $T$, where the relevant theory could be logical, mathematical, conceptual or linguistic according to the context. We believe that, as in the causal case, it

\footnotetext{
${ }^{35}$ E.g. see Schaffer (2016).
} 
is plausible to think of the notions of conceptual explanation and grounding against the background of some theories. This methodological move actually helps to further clarify these notions. It is indeed the theory $T$ that provides the generalizations - in terms of definitions, logical rules, mathematical theorems, conventions and so on - that fix the relations amongst concepts and hence serve to build the hierarchy of conceptual complexity on which conceptual grounding and conceptual explanation rely on. If for example we consider sentence (2) and paragraph (2a), this sentence and paragraph need to be thought of as formulated relative to a geometrical theory $T$, such as Euclid's axiomatic theory. It is $T$ that provides the mathematical definitions of the concepts of triangle, quadrangle, circle, angle and sum, along with the links amongst these concepts. The grounding sentence (2) can be seen as revealing the relationships amongst these concepts fixed by $T$, whilst (2a) is the explanation based on (2), and displaying the generalizations fixed by $T$ that underlie (2). ${ }^{36}$ We now defend this position against two main objections against it.

The first objection relates to the objectivity of grounding: if conceptual grounding and conceptual explanation are relative to theories, then they might no longer seem objective, as is assumed in the current literature (see Section 2 ). The second objection relates to their relation with intuitions: if conceptual grounding and conceptual explanation are relative to theories, then they are not supported by our intuitions as most grounding-theorists claim. ${ }^{37}$ We will answer both these objections at the same time by tapping once again into the parallel with causality-causal explanation.

Consider the causal relation between the knocking of the glass and its fall: at first glance we can say that this relation is objective - it does not depend on any epistemic subject - and that we grasp it in a very intuitive way, namely by simply looking at the glass falling after it has been knocked. However, as we have seen, philosophers ${ }^{38}$ tend to think of causality, as well as of causal explanations, as relative to scientific theories. The causal relation between the knocking of the glass and the falling of the glass is best elaborated relatively to a physical theory containing the law of gravity. Although there might seem to be a contrast between the objectivity and the intuitiveness of the causal relation (and related causal explanation) and the presence of the theory, this contrast turns out to be only apparent. On the one hand, the presence of a theory does not impinge upon the objectivity of the causal relation because the theories we assume to refer to are our best scientific theories, theories which satisfy a certain number of criteria, like adequacy, generality, prediction capacity and so on. ${ }^{39}$ In

\footnotetext{
${ }^{36}$ To take another example, consider the colors red and crimson. We can think of a theory $T$ where the color red is defined as the set of all types of red, namely scarlet, crimson, ...: in $T$ the color red will count as conceptually more complex than the color crimson. However, we can equally think of a theory $T^{\prime}$ where the color red is primitive and crimson is defined as a particular type of red, e.g. red plus violet: in $T^{\prime}$ crimson will count as conceptually more complex than red. Hence, this approach seems to systematize an intricate debate concerning grounding sentences that convey colors, e.g. see (Koslicki, 2015, p. 7).

${ }^{37}$ E.g. see Correia (2014), Fine (2012), Smithson (2020).

${ }^{38}$ See for example Jansson (2015).

${ }^{39}$ See Winther (2016).
} 
other words, the burden of the objectivity is moved from the causal relation to the theory, and, in the context of causality-causal explanation discussions, the theory is assumed to support such a burden. On the other hand, the presence of the theory does not undermine the intuitiveness of the causal relation: on the contrary, the appropriate theory often structures, systematizes and taps into our (correct) intuitions.

Similar points would seem to hold for the case of conceptual grounding and conceptual explanation. Consider the conceptual grounding relation between points and circles in the example with sentences $p$ and $q$ of Section 2: at first glance we can say that this relation is objective - it does not depend on any epistemic subject - and that we grasp it in an intuitive way (probably after having learned a bit of mathematics). However, here we defend the idea that both conceptual grounding and conceptual explanations need to be thought of as relative to theories, such as logical, mathematical, conceptual or linguistic theories. The grounding relation between the points and the circle is for example a relation relative to a geometrical theory where the circle is defined in terms of points. ${ }^{40}$ Although there might seem to be a contrast between the objectivity and the intuitiveness of the grounding relation (and relatedly conceptual explanation) and the presence of the theory, this contrast might be, like in the causal case, only apparent. On the one hand, the presence of a theory does not impinge upon the objectivity of the grounding relation because the theories we assume to work with are our best theories, theories which satisfy a certain number of criteria that lend them some claim to objectivity. ${ }^{41}$ On the other hand, the presence of the theory does not contrast with the intuitiveness of the grounding relation; on the contrary, the theory can be thought of as structuring, systematizing and tapping into our (correct) intuitions. To the extent to which intuitions are correct they will correspond to theories with some objective credentials. The great advantage of moving from intuitions to theories is that these latter provide conceptual grounding and explanations with a rigor and a scientific character that is otherwise absent.

Let us conclude the section with a final remark concerning the examples of conceptual explanations proposed by (Schnieder, 2006, p.403) and provided in the Introduction, namely:

(d) Federica is my sister-in-law because she is married to my brother,

(e) Xanthippe is a widow because Socrates died,

(f) this vase is colored because it is red.

According to our approach, these sentences are examples of conceptual grounding relations, but not of conceptual explanations. ${ }^{42}$ This is so because

\footnotetext{
${ }^{40}$ See also (Rumberg, 2013, p. 443).

${ }^{41}$ E.g. see de Jongh and Betti (2010) or Muddy (2011).

${ }^{42}$ Note that a similar remark applies to the example of causality advocated in Section 2. "There is a fire in the forest because a cigarette was lit in the forest" is a sentence expressing a causal relation. For such a sentence to be a bone fide causal explanation, the specification
} 
there is no mention of the definitions that support the relation between concepts conveyed by (or meaning of expression occurring in) the sentence. In order to count as proper conceptual explanations, they need to be enriched in the following way:

(d') Federica is my sister-in-law because she is married to my brother, via the definition of sister-in-law;

(e') Xanthippe is a widow because Socrates died, according to the definition of widow;

$\left(f^{\prime}\right)$ this vase is colored because it is red, via the characterization of the notion of color as the set of all individual colors.

The main difference between (d)-(f) and (d)'-(f)' is the presence in the latter case of definitions (or charcaterizations) that regulate the connections between the concepts (or meaning of expressions) occurring in the sentences. However, in these cases, insisting on the presence of such definitions may seem overly pernickety. One might even argue that the sentences $(\mathrm{d})^{\prime}-(\mathrm{f})^{\prime}$ do not have a greater explicative force than (d)-(f), and hence doubt that the specification of definitions truly constitutes a distinguishing feature of the notion of explanation. In reply to this objection, note that this impression may be due to the fact that the examples involve concepts (or meaning of expressions), such as sister-in-law or widow, whose definitions and properties are so well-known that they seem hardly worthy of mention. As (Hempel, 1942, p. 361) remarks "In such cases, we feel familiar, at least in a general manner, with the relevant uniform connections $[. .$.$] and thus we are willing to take them for granted without explicit$ mention." In other terms, we are so used to employ the term (or concept) red (or widow or sister-in-law), that we do not feel the need of being reminded of a characterization relating it to other kindred concepts, such color (death, marriage). However, the triviality of the relevant definitions does not imply that they are unnecessary. On the contrary, in more complex examples - mathematical examples are again emblematic - where we do not necessarily master the generalizations (in terms of definitions, theorems...) connecting the concepts (or meanings of expressions) involved, the relevant generalizations stand out as necessary: if they are not specified, we feel that the explanation is incomplete. The more complex examples could be indicative of the inner features of explanations in general and conceptual explanations in this particular case, revealing characteristics that remain hidden in simple cases. Hence, since our aim is to elaborate a general and unifying theory of conceptual explanation and grounding that covers both simple as well as complex cases, we rely on what has been shown in a clear way on more challenging cases. ${ }^{43}$ Adapting (Hempel, 1942, p.

that it is the law of combustion that makes it possible for the cigarette lit in the forest to start the fire, is needed.

${ }^{43}$ While in the philosophy of science literature, the distinction causality-causal explanations relies on the use of generalizations and laws, in the metaphysical literature, as far we know, the distinction metaphysical grounding-metaphysical explanation does not mention such a 
360)'s words on causality to the grounding case, we can claim that an analytic study of explanations cannot content itself with simply registering a grounding relation: it must treat it as material for analysis; it must seek to clarify what is claimed by an explanatory statement of this sort, and how the claim might be supported.

\section{On the formalism for the notions of concep- tual grounding and conceptual explanation}

We have undertaken an informal study of the relationship between the notions of conceptual explanation and conceptual grounding. This section is dedicated to developing a formalism which supports our observations. First we clarify the advantages of a formal approach, and then we present our proposal in detail.

Generally speaking, one could say that one of the best ways to defend any approach is to enfold it within a formalism: ${ }^{44}$ it is the formalism that makes the various features of the approach clear and rigorous. More specifically to our case, the formalism that we will introduce in this Section will clarify two key ingredients of our approach, namely (i) the role that generalizations have in conceptual explanations, and (ii) the precise link between conceptual grounding as a relation and conceptual explanation as a structure. Hence the importance of an appropriate formalism to accompany our approach can hardly be overestimated.

Let us begin by emphasizing a fact that emerges from the literature on conceptual grounding and conceptual explanation. Both these notions have been related, by independent studies, to the notion of proof. On the one hand, several scholars have drawn a parallel between the grounding relation in the Bolzanian tradition and Gerhard Gentzen's cut-free proofs in the sequent calculus. ${ }^{45}$ The parallel mainly rests on the analogy between the fact that a ground is conceptually simpler than its consequence and the fact that the formulas employed to derive the conclusion of a cut-free proof are logically simpler than the conclusion itself. ${ }^{46}$ On the other hand, relying on the Aristotelian distinction between proofs that show that something is true (namely proofs-that), and proofs that show why something is true (namely proofs-why), a long line of thinkers in the philosophy of mathematics, see (Aristotle, 1984, 115, Post. An. I. 2-4), (Leibniz, 1996, Book IV, ch. xvii), (Bolzano, 2004, p. 71), (Frege, 2016, §90), seek to base an account of proofs in mathematics on the primacy of proofs-why. In

feature (an exception is Wilsch (2016)). A conjecture to explain this difference is that the latter literature only considers simple cases and hence the need of the use of generalizations, in metaphysical explanations, does not emerge.

${ }^{44}$ See also (Schaffer, 2016, p.51).

${ }^{45}$ See Buhl (1961); Rumberg (2013); Tatzel (2002).

${ }^{46}$ We remind the reader that a cut-free (logical variant of the) sequent calculus only contains introduction logical rules that introduce formulas either on the right or on the left of the sequent. Hence in a proof $p$ of a cut-free sequent calculus where only logical rules are used, the complexity of the formulas involved in $p$ cannot but increase. For further details see Poggiolesi $(2010,2020)$. 
this perspective, proofs-why are explanatory proofs, i.e. proofs which explain their conclusions by revealing their grounds. Hence they are nothing other than mathematical explanations.

This double reference to the notion of proof, coming independently from grounding and explanation, suggests they are intimately connected to the notion of proof. Inspired by these insights, our aim is to use the notion of proof as a formal background for modeling the contemporary notions of conceptual grounding and conceptual explanation.

To this end, we adopt the standard representation of a proof as a tree according to which the assumptions of the proof are the leaves of the tree, the conclusion of the proof is the root of the tree, and each rule applied in the proof links the nodes corresponding to the premises of the rule to the node corresponding to the conclusion of the rule. Let us list some examples of proofs as tree-objects, where the nodes need to be substituted by sentences that are connected by the corresponding rule, indicated by $r_{i}$.

Example (i)

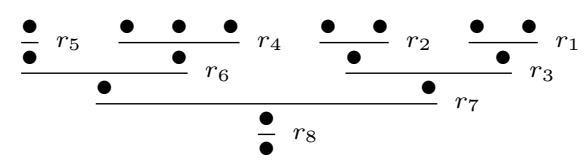

Example (ii)

$$
\begin{array}{ll}
\frac{\bullet}{\bullet} & r_{1} \\
\frac{\bar{\bullet}}{r_{2}} & r_{3} \\
\frac{\bullet}{\bullet} & r_{4} \\
\frac{\bar{\bullet}}{r_{5}} & r_{5}
\end{array}
$$

Example (iii)

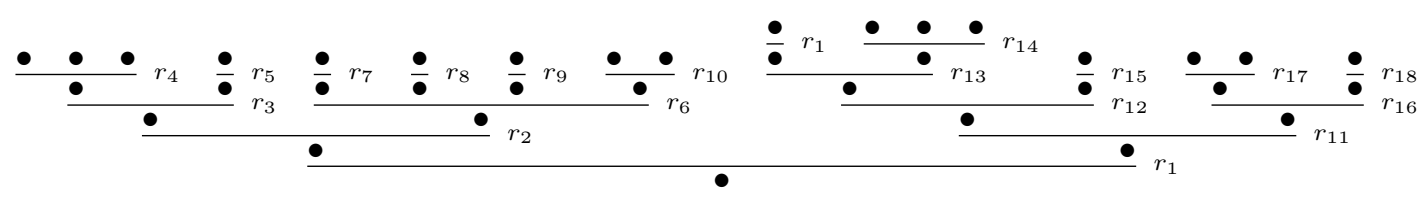

In the case where the nodes are substituted by formulas of a formal language and the $r_{i}$ by appropriate rules, this notion of proof coincides with that of derivation in logic. ${ }^{47}$ Here is an example of a derivation in the natural deduction

\footnotetext{
${ }^{47}$ E.g. see Gentzen (1935); Troelstra and Schwichtenberg (1996).
} 
calculus for classical logic: ${ }^{48}$

$$
\begin{gathered}
\frac{\frac{A \quad B}{A \wedge B} \wedge I}{(A \wedge B) \vee C} \vee I \\
\frac{\neg D \quad((A \wedge B) \vee C) \wedge \neg D}{\neg \neg(((A \wedge B) \vee C) \wedge \neg D)} \neg \neg
\end{gathered}
$$

In this example we have a tree with three leaves, namely the assumptions $A, B$ and $\neg D$; the root of the tree is the formula, $\neg \neg(((A \wedge B) \vee C) \wedge \neg D)$ and each logical rule (namely the rules $\wedge I, \vee I, \neg \neg$ ) applied in the proof links the nodes corresponding to the premises of the rule to the node corresponding to the conclusion of the rule. In these examples of formal proofs, formal systems provide the appropriate rules: indeed, they can be thought of as playing the role of what we have previously called theories. Derivations in logic are but one example of what we mean by proof here: however, theories relevant to conceptual explanation do not have to come in the form of formal systems, nor proofs in the form of derivations.

How can we connect proofs as tree-objects to the notions of conceptual grounding and conceptual explanation? Here is our proposal. We have seen that while conceptual grounding amounts to a relation among sentences, explanations are more refined objects where the generalizations that support the links amongst concepts (or meaning of expressions) need to be specified, and, in this respect, where a division in steps is particularly relevant. But this division in steps implies that conceptual explanations can be profitably thought of as proof-tree-objects: the explanans occupy the leaves of the tree, the explanandum its root, and each step corresponds to the link between the premise(s) and the conclusion of a rule. This simple re-conception, switching from a discursive structure to a hierarchical one, plays a central role in relating conceptual explanations, and not only mathematical explanations, to proof-tree-objects. There is however a second, more subtle ingredient that is required for a full formalization. It concerns the generalizations, in terms of definitions, theorems, conventions and so on, which are characteristic of conceptual explanations and which, in our approach, have the role of supporting the step from explanans and explanandum. How can we reflect and formalize this role in the perspective which sees explanations as proof-tree-objects? It is precisely on this point that this perspective reveals its depth: generalizations correspond to the rules that justify the links between the nodes of the proof-tree; in other words, rules seem to be a perfect and solid way to reflect at the formal level the role that generalizations play in explanations. We first illustrate this point with a simple example; at the end of the section we will set out its advantages. Consider the following conceptual explanation:

\footnotetext{
${ }^{48}$ Because our approach is based on Poggiolesi's formal results (e.g. see Poggiolesi (2016, 2018)) and Poggiolesi uses the natural deduction calculus, we prefer presenting derivations in logic by using the natural deduction logic. However, as underlined before, it is also plausible to use the resources of the sequent calculus.
} 
Federica is my sister-in-law because she is married to my brother, via the definition of sister-in-law.

Suppose one aims at formalizing this conceptual explanation as a proof-tree. We have a two-node proof-tree where the only leaf is the sentence "Federica is married to my brother" and it is linked to the conclusion "Federica is my sister-in law." Namely we have:

\section{Federica is married to my brother}

Federica is my sister-in-law

What is the rule that justifies this passage? And what happens to the definition of 'sister-in-law', which, as we have said in the previous section, is what distinguishes a conceptual explanation from a relation of conceptual grounding? One simple answer to both these questions is the following: the definition of 'sister-in-law' is precisely the rule that justifies the link between premise and conclusion in the proof-tree above. The definition of 'sister-in-law' is usually formulated as the following biconditional:

$\star$ : for any $x$ and $y, x$ is the sister-in-law of $y$ if, and only if, $x$ is married to $y$ 's brother.

However, it can be equivalently conveyed in the form of the following two rules, namely for any $x$ and any $y$ :

$$
\frac{x \text { is the sister-in-law of } y}{x \text { is married to } y \text { 's brother }} s l E \quad \frac{x \text { is married to } y \text { 's brother }}{x \text { is the sister-in-law of } y} s l
$$

The wide and influential literature ${ }^{49}$ on proof-theoretic semantics supports precisely the view that definitions can be conceived as and conveyed by means of rules. It has also been shown ${ }^{50}$ that defining a term (or concept) prooftheoretically - that is, via rules - is actually equivalent to defining it via the corresponding biconditional. Hence, the rules $s l I$ and $s l E$ can be taken to define the term (or concept) 'sister-in law.' Note the difference between these two rules: whilst slE removes or eliminates the notion of 'sister-in-law' if read from top to the bottom, slI introduces this term (or concept). Indeed, while the former is called an elimination rule, the latter, which introduces a new term (or concept), is an introduction rule. ${ }^{51}$ The introduction rule slI can thus be used to explain a sentence containing the term 'sister-in-law' by one containing the simpler terms 'married to' and 'brother.' But then, it is easy to see that it is precisely thanks to this rule that the passage from "Federica is married to my

\footnotetext{
${ }^{49}$ E.g. see Francez (2019); Schroeder-Heister (2018, 1991).

${ }^{50}$ E.g. Sambin et al. (2000).

${ }^{51}$ On the logical difference between introduction and elimination rules see Troelstra and Schwichtenberg (1996).
} 
brother" to "Federica is my sister-in law" is justified: it is an instance of the rule.

It thus seems that by moving to a hierarchical structure, and by thinking of generalizations as rules, proof-tree-objects can be profitably used to model conceptual explanations. Crucially, not any proof-tree object represents a 'proper' conceptual explanation, but only those involving an increase in conceptual complexity from the premise(s) to the conclusion. This kind of proof-tree object has been discussed in the tradition, see for instance Betti (2010); Mancosu (1999); Sebestik (1992). According to Rumberg (2013), there exists a stringent parallel between this particular kind of proof and the so-called canonical normal derivations in intuitionistic logic, e.g. see Prawitz (1965). Poggiolesi (2018) formalizes the counterpart of proofs as tree-objects for logical explanation with her concept of formal explanations. Formal explanations are composed of introduction rules and involve the appropriate complexity increase for logical explanations. As such, they can be seen as the skeleton underlying (our conception of) conceptual explanations, though set out for the special case of logical explanation. The reader interested in further technical detail concerning our idea of proof-tree is referred to Poggiolesi (2018).

We have introduced a conception of explanation in terms of a particular type of proof-tree object, i.e. a proof-tree object with a conceptual complexity increase from top to bottom. What does conceptual grounding correspond to in this framework? Based on our discussion in Section 3, but also according to Poggiolesi's formal work, it seems reasonable to take conceptual grounding as the relation between appropriate premise(s) and conclusions occurring in the conceptual explanation proof-tree. In particular, we have a relation of immediate conceptual grounding when the premise(s) and conclusion are directly linked in the relevant proof-tree; we obtain a relation of mediate conceptual grounding by taking premises and conclusion separated by several consecutive steps (i.e. a path) in the proof-tree. Under this proposal, proof-trees neatly formalize the relationship between conceptual explanation and conceptual grounding: the former are complex hierarchical structures made of nodes representing premises and conclusions which are linked according to (introduction) rules; the latter conveys a relation amongst the (appropriately selected) elements of these structures, but where no explicit mention of rules is necessary.

This proof-based perspective on conceptual explanation-conceptual grounding emerges and copes comfortably with the more complex examples. Let us illustrate this point on an example coming from logic, as well as the example discussed in Section 3. Consider again the following derivation:

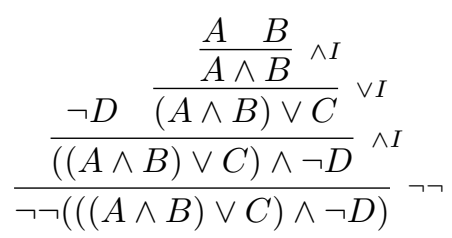


Figure 1: Quadrangle ABCD

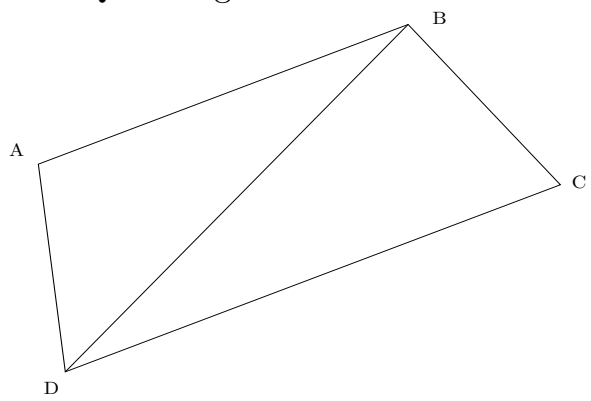

On the one hand, this peculiar derivation, which displays a complexity increase from the premisses to the conclusion, can be seen as a logical and hence conceptual explanation that has as explanans the formulas $A, B$ and $\neg D$ and as explanandum the formula $\neg \neg(((A \wedge B) \vee C) \wedge \neg D)$ : in this explanation each step is specified together with the introduction rule that justifies the step. On the other hand, also thanks to this derivation, we can identify a logical (and hence conceptual) mediate grounding relation between the formulas $A, B, \neg D$ and the formula $\neg \neg(((A \wedge B) \vee C) \wedge \neg D)$. In other words, a conceptual grounding relation is the relation amongst (appropriate) formulas occurring in the structure of the corresponding conceptual explanations.

Let us now move to considering example (2a), which, recall, is the following explanation:

(2a) The reason why the sum of the four angles of any quadrangle is equal to a full circle is that the sum of four angles of any quadrangle is equal to the sum of four right angles, via definition (i) that says that any full circle corresponds to four right angles.The reason why the sum of the four angles of any quadrangle is equal to four right angles is that the sum of the three angles of a triangle is equal to two right angles, via theorem (ii) that says that any quadrangle can be divided into two triangles whose angles taken together are equal to the angles of the quadrangle.

Putting (2a) in the form of a proof-tree, we obtain the following object. For any four points $\mathrm{ABCD}:^{52}$

\footnotetext{
${ }^{52}$ In the interests of readability, we use the following abbreviations:

- $\mathrm{T}$ for triangle,

- $Q$ for quadrangle,

- $2 R A$ for two right angles,

- $4 R A$ for four right angles.
} 
if $\mathrm{ABD}$ is a $\mathrm{T}$, then the sum of its angles $=2 \mathrm{RA} \quad$ if $\mathrm{BDC}$ is a $\mathrm{T}$, then the sum of its angles $=2 \mathrm{RA}$

$$
\frac{\text { if ABCD is a } Q \text {, then the sum of its angles = 4RA }}{\text { if ABCD is a } Q \text {, then the sum of its angles }=\text { full circle }} r_{2}
$$

where $r_{1}$ is the rule that corresponds to theorem (i), namely the rule, for any four points ABCD:

if $\mathrm{ABD}$ is a $\mathrm{T}$, then the sum of its angles $=z \quad$ if $\mathrm{BDC}$ is a $\mathrm{T}$, then the sum of its angles $=x$

if $\mathrm{ABCD}$ is a $\mathrm{Q}$, then the sum of its angles $=z+x$

whilst $r_{2}$ is the rule that corresponds to definition (ii), namely the rule, for all $x$,

$$
\frac{x=\text { four right angles }}{x=\text { full circle }}
$$

So the conceptual explanation of why the sum of the four angles of a quadrangle is equal to a full circle has the form of the proof-tree-object above constructed in a theory encompassing at least the rules mentioned, and involving an increase of conceptual complexity from the top to the bottom (at each step a new concept, defined in terms of the previous ones, is introduced). Given this, we can claim that there is a conceptual grounding relation, relative to the same theory, between the premise of the conceptual explanation (the sum of the three angles of a triangle is equal to two right angles) and its conclusion (the sum of the four angles of any quadrangle is equal to a full circle). This relation of conceptual grounding is mediate since it involves several consecutive steps. Moreover, even in the conceptual grounding relation, the consequence is conceptually more complex than the grounds and this provides the directionality of the relation. However, in the grounding relation, since it is precisely a relation and not an explanation, there is no need to specify the rules that support the relation itself, nor to display the details of the connecting structure.

We would like to end the section by dwelling on two important points relative to the formalism that has been introduced in this section. The first concerns the formalization of generalizations in conceptual explanations as rules supporting steps of proof-trees. Although we have already discussed this point in the previous section, we think it is worth to bring further arguments in its defense by confronting it with the more famous and widespread DN-model, where generalizations occur in explanations as part of the explanans.

The first advantage of our way of treating generalizations in proof-trees is that it ensures the conceptual complexity increase from the premisses to the conclusion. Following the model of Poggiolesi (2018), only generalizations as introduction rules are allowed in our proof-trees; but then, since introduction rules do nothing but introducing a new concept at a time, then the conceptual complexity cannot but increase going from the premisses to the conclusion in our proof-trees. This is in stark contrast with the DN-model, or any other 
approach which uses the generalizations as premisses of the proof-tree, because the step from a generalization to one of its instances does not involve an increase of conceptual complexity, but rather a complexity decrease, if anything. On the basis of these considerations, the use of generalizations as (introduction) rules and the conceptual complexity increase ${ }^{53}$ in conceptual explanations emerge as two faces of the same medal: having embraced the defense of the latter from the literature, we cannot but put forward an approach that relies on the former.

The second advantage of using generalizations as rules and not as premisses of the explanations is that this is precisely what protects our approach from counterexamples analogous to those of the DN-model. Recall that, according to the DN-model, an explanation is any object that displays the following structure:

$$
\begin{aligned}
& \mathrm{S} \text { and } \mathrm{K}_{1}, \ldots \mathrm{K}_{n} \text {, then } T \\
& \mathrm{~S} \text { and } \mathrm{K}_{1}, \ldots \mathrm{K}_{n}
\end{aligned}
$$

$\mathrm{T}$

All counterexamples to this model work by substituting the $T$ with the $S$ and vice-versa and showing that the inference still holds. When this is the case, the asymmetry of explanations is betrayed: if the premisses explain the conclusion, it cannot be the case that the conclusion explains the premisses. In our approach this type of counterexample can never arise. Since we use generalizations as introduction rules, it is not possible to invert the conclusion with the premisses and still have an inference compatible with our proof-tree objects. Hence, the use of generalizations as (introduction) rules means that our approach does not suffer from the standard counterexamples raised against the DN-model.

Let us now turn to the second point concerning our formalism. Despite the advantages discussed above, one might still wonder whether another formal framework could do a better job. In particular, one might look at the structural equational framework, which has been famously used by Woodward (2003) to formalize the notion of causal explanation, but also more recently by Schaffer (2016) to model the notion of metaphysical grounding: could the structural equation framework be used to capture the notions of conceptual grounding and explanation as well? Ultimately, of course, a full answer will have to wait for an attempt to work out such a structural-equations-based approach to conceptual grounding. However, we shall venture some remarks that do not speak in its favor. First of all, it is important to once more underlying that conceptual explanations include mathematical explanations. It seems hard to see how the structural equation framework could model mathematical explanations better than proof-tree objects given that these explanations are nothing but particu-

\footnotetext{
${ }^{53}$ Here conceptual complexity should be understood in terms of composition of concepts. As shown by Ginammi et al. (2020), if conceptual complexity is understood in terms of generality, then it is a requirement compatible with the presence of generalizations as premisses in explanations.
} 
lar types of proofs. Moreover, the proof-tree objects proposed here, which are based on formal explanations put forward by Poggiolesi (2018), are finite objects that naturally display a hierarchical structure, which is invariably related to complexity. In particular, this type of proof does not allow for loops; rather they reduce, via a step-by-step procedure, the conceptual complexity from the conclusion to the premise(s) until some atomic concepts (or meaning of expressions) are reached. On this front, they are perfectly adapted to representing conceptual explanations, namely explanations where one descends from a sentence to its reasons through a hierarchy of (decreasing) complexity levels that eventually stops when no further reduction is possible. The structural equation framework is much more liberal; it allows for loops and do not necessarily display any hierarchy. Hence, in the absence of particular restrictions (which remain to be worked out), whilst the structural equations framework works well for the causal case, and it has also been applied to metaphysics, proofs as tree-objects seem naturally better equipped for the conceptual domain.

\section{Conclusions}

Following the example of philosophy of science where the causality-causal explanation pair is a major focus of research, but also looking at metaphysics where there is a new trend dealing with metaphysical grounding in its relationship to metaphysical explanation, this paper has studied the couple conceptual grounding-conceptual explanation. In particular we have analyzed the relationship between the two notions, and we have elaborated a proof-based formal framework to capture these notions and their relation. We think that this study suggests at least two promising lines of research. On the one hand, we think that it would be interesting to use the recent results in proof theory to better investigate the inner structure of conceptual explanations and to develop the connection between this kind of explanation and the literature on logic and philosophy of mathematics. On the other hand, our research opens up the question of the relationship between the three pairs of notions causality-causal explanations, metaphysical grounding-metaphysical explanation, and conceptual grounding-conceptual-explanations. The comparison between them could be undertaken both at the conceptual level and at the formal level, given that formal frameworks have been developed for each pair.

\section{References}

Aristotle (1984). The Complete Works of Aristotle. Princeton University Press.

Arnauld, A. and Nicole, P. (1970). La Logique ou l'art de penser. Flammarion.

Audi, P. (2012). Grounding: Toward a theory of the in-virtue-of relation. Journal of Philosophy, 109:658-711. 
Betti, A. (2010). Explanation in metaphysics and Bolzano's theory of ground and consequence. Logique et analyse, 211:281-316.

Bolzano, B. (1996). Contributions to a more well founded presentation of mathematics. In Ewald, W. B., editor, From Kant to Hilbert : A source book in the foundations of mathematics, pages 176-224. Oxford University Press, Oxford.

Bolzano, B. (2004). On the Mathematical Method and Correspondence with Exner. Rodopi.

Bolzano, B. (2015). Theory of Science. Oxford University Press, Oxford.

Buhl, G. (1961). Ableitbarkeit und abfolge in der wissenschaftslehre bolzanos. In Heidemann, I., editor, Kantstudien Ergänzungshefte, 83, pages 176-224. Kölner Univer- sitätsverlag.

Carrara, M. and De Florio, C. (2020). Identity criteria: An epistemic path to conceptual grounding. Synthese, forthcoming:1-19.

Chalmers, D. (2012). Constructing the world. Oxford University Press.

Correia, F. (2014). Logical grounds. Review of Symbolic Logic, 7(1):31-59.

Correia, F. and Schnieder, B. (2012). Grounding: an opinionated introduction. In Correia, F. and Schnieder, B., editors, Metaphysical grounding, pages 1-36. Cambridge University Press, Cambridge.

de Jongh, W. and Betti, A. (2010). The classical model of science - a millenniaold model of scientific rationality. Synthese, 174:185-203.

Detlefsen, M. (1988). Fregean hierarchies and mathematical explanation. International Studies in the Philosophy of Science, 3:97 - 116.

Fine, K. (2012). Guide to ground. In Correia, F. and Schnieder, B., editors, Metaphysical grounding, pages 37-80. Cambridge University Press, Cambridge.

Francez, N. (2019). Relevant connexive logics. Logic and Logical Philosophy, 30:1-18.

Frege, G. (2016). The basic laws of arithmetic, 1-2. Oxford University Press.

Gentzen, G. (1935). Untersuchungen über das logische schließen. Mathematische Zeitschrift, 39:176-210.

Ginammi, A., Koopman, R., Wang, S., Bloem, S., and Betti, A. (2020). Bolzano, Kant, and the traditional theory of concepts, a computational investigation. In Press, P. U., editor, The Dynamics of Science: Computational Frontiers in History and Philosophy of Science, pages 1-36. de Block, A. and Rams, G.

Glaizer, M. (2020). Explanation. In Raven, M., editor, The Routledge Handbook of Metaphysical Grounding, pages 215-225. Routledge. 
Hempel, C. (1942). The function of general laws in history. Journal of Philosophy, 39:35-48.

Hofweber, T. (2009). Ambitious, yet modest, metaphysics. In Chalmers, D., Manley, D., and Wasserman, R., editors, Metamteaphysics: New Essays on the Foundations of Ontology, pages 260-289. Clarendon Press, Oxford.

Jansson, L. (2015). Explanatory asymmetries: Laws of nature rehabilitated. Journal of Philosophy, 86:45-63.

Jansson, L. (2017). Explanatory asymmetries, ground, and ontological dependence. Erkenntnis, 82:17-44.

Kitcher, P. (1981). Explanatory unification. Philosophy of Science, 48(4):507531.

Koslicki, K. (2015). The coarse-grainedness of grounding. Oxford Studies in Metaphysics.

Leibniz, W. (1996). New Essays on Human Understanding. Cambridge University Press.

Mancosu, P. (1999). Bolzano and Cournot on mathematical explanation. Revue d'histoire des sciences, 52(3):429-456.

Mancosu, P. (2001). Mathematical explanation: Problems and prospects. Topoi, 20(1):97-117.

Mancosu, P. (2008). Mathematical explanations : Why it matters. In Mancosu, P., editor, The Philosophy of Mathematical Practice. OUP Oxford.

Maurin, A.-S. (2019). Grounding and metaphysical explanation: it's complicated. Philosophical Studies, 176:1573 - 1594.

Menzies, P. and Beebee, H. (2019). Counterfactual theories of causation. In Zalta, E. N., editor, The Stanford Encyclopedia of Philosophy, pages 1-45. Stanford.

Merlo, G. (2020). Disjunction and the logic of grounding. Erkenntnis, 30:1-24.

Muddy, P. (2011). Defending the Axioms. OUP, Oxford.

Paoli, F. (1991). Bolzano e le dimostrazioni matematiche. Rivista di Filosofia, LXXXIII:221-242.

Pascal, B. (1993). De l'esprit géométrique. Gallimard.

Poggiolesi, F. (2010). Gentzen calculi for modal propositional logic. Springer.

Poggiolesi, F. (2016). On defining the notion of complete and immediate formal grounding. Synthese, 193:3147-3167. 
Poggiolesi, F. (2018). On constructing a logic for the notion of complete and immediate formal grounding. Synthese, 195:1231-1254.

Poggiolesi, F. (2020). A proof-based framework to several types of grounding. Logique et Analyse, pages 1-32.

Prawitz, D. (1965). Natural deduction. A proof theoretic study. Almquist and Wiskell.

Prawitz, D. (2019). The concept of proof and ground. In Centrone, S., Negri, S., and Schuster, P., editors, Mathesis Universalis, Computability and Proof, pages 290-309. Springer.

Raven, M. (2012). In defense of ground. Australasian Journal of Philosophy, 90:687-701.

Raven, M. (2015). Ground. Philosophy Compass, 10:322-333.

Roski, S. and Rumberg, A. (2016). Simplicity and economy in Bolzano's theory of grounding. Journal of the History of Philosophy, 54:469-496.

Roski, S. and Schnieder, B. (2019). Fundamental truths and the principle of sufficient reason in Bolzano's theory of grounding. Journal of the History of Philosophy, 57:675-706.

Rumberg, A. (2013). Bolzano's theory of grounding against the background of normal proofs. Review of Symbolic Logic, 6(3):424-459.

Salmon, W. (1984). Explanation and the Causal Structure of the World. Princeton University Press.

Sambin, G., Battilotti, G., and Faggian, C. (2000). Basic logic: Reflection, symmetry, visibility. Journal of Symbolic Logic, 92:979-1013.

Schaffer, J. (2016). Grounding in the image of causation. Philosophical Studies, $173: 49-100$.

Schnieder, B. (2006). A certain kind of trinity: Dependence, substance, and explanation. Philosophical Studies, 129:393 - 419.

Schnieder, B. (2011). A logic for 'Because'. The Review of Symbolic Logic, $4(03): 445-465$.

Schroeder-Heister, P. (1991). Uniform proof-theoretic semantics for logical constants (abstract). Journal of Symbolic Logic, 56:11-42.

Schroeder-Heister, P. (2018). Proof-theoretic semantics. In Zalta, E., editor, The Stanford Encyclopedia of Philosophy, pages 1-45. Stanford.

Sebestik, J. (1992). Logique et mathematique chez Bernard Bolzano. J. Vrin. 
Smithson, R. (2020). Metaphysical and conceptual grounding. Erkenntnis, forthcoming:1-25.

Steiner, M. (1978). Mathematical explanation. Philosophical Studies, 34(2):135151.

Strevens, M. (2008). Depth: An Account Of Scientific Explanation. Harvard University Press.

Tahko, T. (2018). Fundamentality. In Zalta, E., editor, The Stanford Encyclopedia of Philosophy, pages 1-45. Stanford.

Tatzel, A. (2002). Bolzano's theory of ground and consequence. Notre Dame Journal of Formal Logic, 43(1):1-25.

Troelstra, A. S. and Schwichtenberg, H. (1996). Basic Proof Theory. Cambridge University Press.

Williamson, T. (2007). The Philosophy of Philosophy. Blackwell.

Wilsch, T. (2016). The deductive-nomological account of metaphysical explanation. Australasian Journal of Philosophy, 94:1-23.

Winther, R. (2016). The structure of scientific theories. In Zalta, E., editor, The Stanford Encyclopedia of Philosophy, pages 1-32. Stanford Education.

Woodward, J. (2003). Making Things Happen: A Theory of Causal Explanation. Oxford University Press.

Woodward, J. (2019). Scientific explanation. In Zalta, E. N., editor, The Stanford Encyclopedia of Philosophy, pages 1-43. Stanford. 